\title{
INICIATIVAS DE CLÚSTER \\ PARA EL FORTALECIMIENTO \\ DE LA COMPETITIVIDAD \\ EN EL DEPARTAMENTO DE \\ NORTE DE SANTANDER: \\ CASOS DE TURISMO DE \\ SALUD Y SISTEMA MODA
}

\section{RESUMEN}

Este trabajo estudia las políticas clúster y su configuración como iniciativas planificadas, centrándose en el análisis del Programa de Rutas Competitivas en Colombia. Se utiliza una metodología cualitativa para investigar el rol de los actores del clúster como elementos fundamentales del Programa. Los resultados de la investigación están relacionados con el proceso de configuración de clústeres, los factores favorables y limitantes de su actividad, la valoración general del programa y la identificación de áreas de mejora. A través de la iniciativa Rutas Competitivas, que bajo la coordinación de la Cámara de Comercio de Cúcuta y la firma consultora Competitiveness, se organizó la estrategia de clúster para fortalecer la competitividad de sectores relevantes de la economía nortesantandereana, tales como el turismo de salud mediante la iniciativa Mi Destino Salud, y el de confecciones y marroquinería, con la iniciativa del Sistema Moda.

Palabras clave: Clúster, Turismo de salud, Sistema Moda, Competitividad, Benchmarking. 


\section{CLUSTER INITIATIVES TO STRENGTHEN THE COMPETITIVENESS IN NORTE DE SANTANDER: HEALTH TOURISM AND FASHION SYSTEM}

\section{ABSTRACT}

This paper is part of the case study in the application of policies and cluster configuration and planned initiatives, focusing on the analysis of the program Rutas Competitivas in Colombia. A qualitative methodology is used to investigate the role of cluster actors as key elements of the program. The research results are related to the cluster setup process, the favorable factors and those limiting its activity, the overall assessment of the program and identification of improvement areas. Through the Rutas Competitivas initiative, coordinated by the Chamber of Commerce of Cúcuta and the consulting firm Competitiveness, cluster strategy was organized to strengthen the competitiveness of major sectors of Norte de Santander, such as health tourism through Mi Destino Salud Initiative, and the apparel and leather goods, with the initiative Sistema Moda.

Keywords: Cluster, Health Tourism, Fashion System, Competitiveness, Benchmarking.

\section{INTRODUCCIÓN}

Durante los últimos años, la coyuntura económica de Norte de Santander se ha caracterizado por un entorno de recesión económica que se ha agudizado por el desempeño de la economía venezolana y la caída del precio del bolívar fuerte frente al peso, lo cual resta competitividad a los precios de los productos y servicios en Colombia. Adicionalmente, el cierre indefinido de la frontera colombo-venezolana ha reforzado la necesidad de reducir considerablemente la dependencia de la actividad económica del departamento con Venezuela.

Ante este escenario, surge la necesidad de plantear una reconversión de la estructura económica de la región, para generar un ambiente propicio a las inversiones y explorar nuevos mercados para impulsar el desarrollo empresarial. Así, el reto del departamento es estructurar y consolidar una base económica estable y menos vulnerable a factores ligados a la coyuntura política, social y económica de Venezuela.

Inicialmente, es lógico que romper los lazos históricos no es una labor sencilla, ni de corto plazo. Por el contrario, se debe concebir como un proceso de transformación productiva
* Magís ter, Profesor investigador. Fundación de Estudios Superiores Comfanorte FESC.Colombia. Correo-e: j_corredor@fesc.edu.co

Recibido: 26 de enero de 2017, aceptado: 27 de mayo de 2017.

Para citar el artículo: Corredor, J. (2017). "Iniciativas de clúster para el fortalecimiento de la competitividad en el departamento de Norte de Santander: casos de turismo de salud y sistema moda", en: Sotavento MBA, n. ${ }^{\circ} 29$, pp. 54-66. D0I: http://dx.doi.org/10.18601/01233734.n29.06 
para Norte de Santander, el cual requiere de un cambio de mentalidad que va desde los ciudadanos hasta los empresarios, y exigiendo un rol preponderante de las instituciones académicas.

Las iniciativas de Mi Destino Salud y Sistema Moda surgen en el marco del programa de Rutas Competitivas del Ministerio de Comercio, Industria y Turismo, en alianza con la Cámara de Comercio de Cúcuta, Innpulsa, la Comisión Regional de Competitividad de Norte de Santander y las instituciones de educación superior Universidad de Santander - UDES y la Fundación de Estudios Superiores Comfanorte - FESC, con el propósito de generar propuestas encaminadas a transformar los sectores de turismo de salud y de confecciones y marroquinería de Norte de Santander, e incrementar su productividad basándose en estrategias, acciones y políticas de índole local y nacional.

Dada la pertinencia y el nivel de experiencia en las diferentes áreas, la UDES y la FESC fueron aliadas importantes durante el proceso de estructuración de las iniciativas, participando la primera en Mi Destino Salud, y la segunda en el Sistema Moda. Ambas instituciones participaron como socios de las iniciativas con el fin de fortalecer el proceso desde la perspectiva de la academia.

El desarrollo de la iniciativa tuvo la asesoría metodológica de la firma española Competitiveness, la cual se especializa en generar alianzas público-privadas para mejorar la competitividad de las empresas de una región. Esta metodología ha sido probada en más de 200 proyectos, en más de 40 sectores económicos diferentes de 30 regiones de diversas partes del mundo.

Bajo el programa de Rutas Competitivas se inició un proceso para impulsar el desarrollo regional y promover la construcción de capacidades locales para el análisis y fortalecimiento de clústeres.

Para el caso de Norte de Santander, la iniciativa se ha convertido en un reto de alta exigencia, debido a que el proceso de fortalecimiento de un clúster requiere cambiar la mentalidad de los actores del mismo. Esto generalmente va en dirección contraria a los preceptos tradicionales sobre desarrollo y competitividad.

La estructuración de las iniciativas tiene tres fases: identificar los desafíos, guiar la estrategia y lanzar las acciones.

\section{FUNDAMENTO TEÓRICO}

Los elementos conceptuales de clúster y competitividad tienen una relación directa.

\subsection{CLÚSTER}

Antes de profundizar en el proceso de fortalecimiento de los clústeres de turismo de salud y Sistema Moda en Norte de Santander, es pertinente abordar los conceptos esenciales sobre clúster.

Es importante considerar que, según Gómez (1999), el proceso de conformación de un clúster en una actividad económica en particular requiere en primera instancia de una adecuada comprensión de la visión del negocio, de sus integrantes y del entorno en que se desenvuelve. Esto significa que aunque se Ilegue con una propuesta convincente, si los beneficiarios de la propuesta no la comprenden en su justa dimensión, o las condiciones en que se desempeñan son precarias, el tiempo y esfuerzo dedicado a la iniciativa no serán los deseables.

Para Porter (2008), un clúster se define como la concentración (natural) geográfica de empresas interconectadas, proveedores especializados, proveedores de servicios, empresas en sectores relacionados e instituciones asociadas (como universidades, agencias de estándares y gremios) que compiten pero también cooperan.

En la actual dinámica de la competitividad, los clústeres representan, por un lado, una 
nueva forma de organización de la cadena de valor y, por otro, nuevas formas de jerarquías organizacionales o integración vertical. La proximidad geográfica de compañías e instituciones y el establecimiento de relaciones entre ellas procuran una mayor coordinación y confianza que la simple interacción de mercado entre actores dispersos geográficamente.

Esta coordinación y confianza entre organizaciones es mucho más flexible que la que provee las integraciones verticales o las relaciones formales entre empresas como redes, alianzas o colaboraciones.

Desde la perspectiva del turismo, Jaramillo (2003) define un clúster turístico como un espacio geográfico determinado por unas ventajas comparativas concretas, lo bastante homogéneas, territorialmente enmarcadas en una relativa unidad y con unas condiciones de conectividad promisorias.

Así mismo, existen atractivos naturales - culturales necesarios para perfilar un buen producto turístico o una familia de productos, más los componentes empresariales y tecnológicos que forman una cadena de producción y mercadeo armónica y relativamente autárquica, la cual debe operar en condiciones adecuadas de productividad, para ofrecer el producto o productos en condiciones de excelencia, a menor precio que los competidores de calidad comparable, y en la oportunidad y cantidad buscadas por la demanda. Es decir, donde las ventajas comparativas se puedan maximizar como ventajas competitivas.

En el caso de la moda, el impacto de la sociedad de la información, el uso intensivo de las tecnologías de la información y la aparición de nuevos comportamientos empresariales y sociales han alterado profundamente el modo en que se produce, distribuye, comercializa y vende la moda. Todos estos cambios configuran un nuevo sistema en el que la información y el tiempo se convierten en factores clave de la relación entre la empresa y el cliente, un proceso complejo que debe culminar en el acto de producir y poner a su disposición a tiempo.

En la industria de la moda, se pueden distinguir cuatro modelos de producción (Nueno, 2001). El primero, el tradicional, necesita de un ciclo de más de 300 días desde que se diseña un modelo, se hacen los patrones, se encarga el tejido, se fabrica y se procede a su distribución. Un segundo modelo sería un híbrido, en tanto sigue la pauta del tradicional, aunque incorpora unas producciones más cortas y más flexibles que garantizan la rotación de los productos en las tiendas. El tercer modelo es el que pasa a repartir su producción entre las prendas "básicas", aquellas que se considera que no pasan de moda y, por tanto, tienen una baja rotación en las tiendas y las prendas "oportunistas" o just in time, que son susceptibles de rotar en ciclos cortos atendiendo a las variaciones del mercado.

Por ejemplo, la empresa Zara ha reducido los tiempos en dos semanas, tras haber desarrollado un modelo de producción en red a partir de la interconectividad de un amplio número de pequeñas y medianas empresas encargadas de la producción, distribución y venta con las que la casa matriz mantiene lazos basados en la subcontratación y en su caso en la franquicia. Pero, a diferencia de sus competidores, Zara ha sido la primera empresa que ha incorporado todo un sistema basado en Internet, que le permite facilitar los flujos de información entre los más de 2.244 puntos de venta que tiene repartidos en 58 países.

En general, todo esto ha llevado a reconfigurar los esquemas de producción y prestación de servicios, para adaptar las capacidades de las empresas a las nuevas exigencias de los mercados internacionales mediante un nuevo modelo de empresa donde la información y el tiempo se convierten en factores claves en la relación con el cliente. 


\subsection{COMPETITIVIDAD}

El valor agregado de la clusterización es la capacidad de generar beneficios colectivos en un entorno de cooperación, pero que coexiste con la competencia. En este sentido, surge el concepto de la 'coopetencia'. De acuerdo con Nalebuff y Brandenburger (1996), este término integra la tensión y dinámica estratégica que impulsa una empresa para combinar, de manera complementaria, procesos de cooperación y competencia.

Para Altvater y Mahnkop (2002), el proceso que explica por qué coexisten cooperación y competencia dentro y entre empresas es el siguiente: el incremento de innovaciones tecnológicas en las firmas, si bien aumenta productividad y permite enfrentar la competencia, también eleva la composición orgánica del capital y, por tanto, reduce la tasa de ganancia. Esta caída en rentabilidad, paradójicamente, ha contribuido a reforzar lazos de cooperación y fusiones empresariales, más allá de fronteras nacionales.

En un entorno empresarial como el actual, que se caracteriza por la acelerada competencia, las relaciones o vínculos interorganizacionales se convierten en un factor estratégico para las empresas, que puede generar grandes sinergias y beneficios tales como mejorar la competitividad de las empresas mediante la cooperación; focalizar los retos estratégicos competitivos que no pueden abordarse mediante acciones individuales de las empresas; optimizar los costos mediante la implementación de economías de escala, y aumentar el poder de negociación a nivel sectorial.

\section{METODOLOGÍA}

Como se enunció previamente, la metodología se fundamenta en la transferencia de conocimiento otorgada por la firma consultora
Competitiveness, con domicilio en Barcelona, España. Bajo las directrices dadas, el proceso de fortalecimiento de clústeres se lleva a cabo en tres fases: i) identificar los desafíos, ii) guiar la estrategia y iii) lanzar las acciones. A continuación, se explica el desarrollo metodológico de cada una de las fases.

\subsection{IDENTIFICAR LOS DESAFÍOS}

En la primera fase, el proceso de organización de los clústeres de turismo de salud y el Sistema Moda busca entender quiénes son los agentes locales del territorio, cómo se organizan, cuáles son sus fuerzas y debilidades, y detectar a qué retos se enfrentan las empresas. La principal actividad de esta etapa es realizar entrevistas a los actores claves del clúster y estudiar los documentos e investigaciones existentes sobre la industria a nivel internacional, nacional y local.

Este ejercicio es relevante porque permite conocer la dinámica del sector y comprender las variables que influyen en el comportamiento de los agentes que participan en el mismo.

\subsection{GUIAR LA ESTRATEGIA}

Durante esta etapa se define, conjuntamente, cuáles son las opciones estratégicas más interesantes para la tipología de empresas presentes en el clúster, siempre en función de la evolución de las tendencias del mercado y de la demanda. Para alcanzar este propósito, se realizan entrevistas con compradores sofisticados y un viaje de estudio a regiones de referencia para llevar a cabo un proceso de benchmarking. Además, se convoca a diferentes actores claves del clúster para conformar grupos de reflexión estratégica con las empresas, a quienes se les comparte las experiencias registradas en el viaje de referencia.

Esta etapa tiene como objetivo validar la información obtenida en la revisión documental 
y generar las bases para el diseño de una estrategia competitiva para el clúster. El valor agregado del ejercicio es el aporte realizado por los empresarios y demás actores del sector, de tal forma que se les involucra directamente en el proceso de formulación de la planeación estratégica del clúster.

\subsection{LANZAR LAS ACCIONES}

La última etapa del proceso de fortalecimiento del clúster consiste en poner en marcha grupos de trabajo (formados por las empresas y entidades locales), con el propósito de llevar a la práctica la visión estratégica desarrollada. Esto tiene como principal objetivo que los mismos actores del clúster sean quienes generen acciones puntuales para impulsar el sector.

Las tres fases se enmarcan en el siguiente gráfico, que resume el proceso descrito anteriormente.

Cabe resaltar que al término de cada una de las fases se desarrollan reuniones de socialización con los actores interesados para exponer los avances del proceso de fortalecimiento de la competitividad, el cual tiene una duración aproximada de ocho meses.
La idea central bajo la cual las iniciativas de clúster fueron establecidas es que no sean excluyentes, pero para efectos de la obtención de mejores resultados es necesario filtrar la cantidad de empresas que pueden participar en el desarrollo de las acciones. Esto tiene como elemento diferenciador la experiencia, la calidad, la tecnología y la infraestructura de los prestadores de los servicios, para que sean las empresas que lideren el proceso de cambio y desarrollo del sector, así como el potencial de crecimiento de las empresas, fundamentados en la capacidad de adaptarse a la mentalidad de cambiar de manera sustancial la forma de hacer sus negocios.

\section{RESULTADOS}

Considerando las fases de implementación de la iniciativa, luego de realizar las actividades en la primera etapa, se identificaron desafíos, tales como la necesidad de reemplazar el mercado natural que representa Venezuela, desarrollar un medio que promueva el trabajo entre los diferentes agentes del clúster, acercar la academia y el sector productivo para analizar

Gráfico 1. Iniciativa Mi Destino Salud

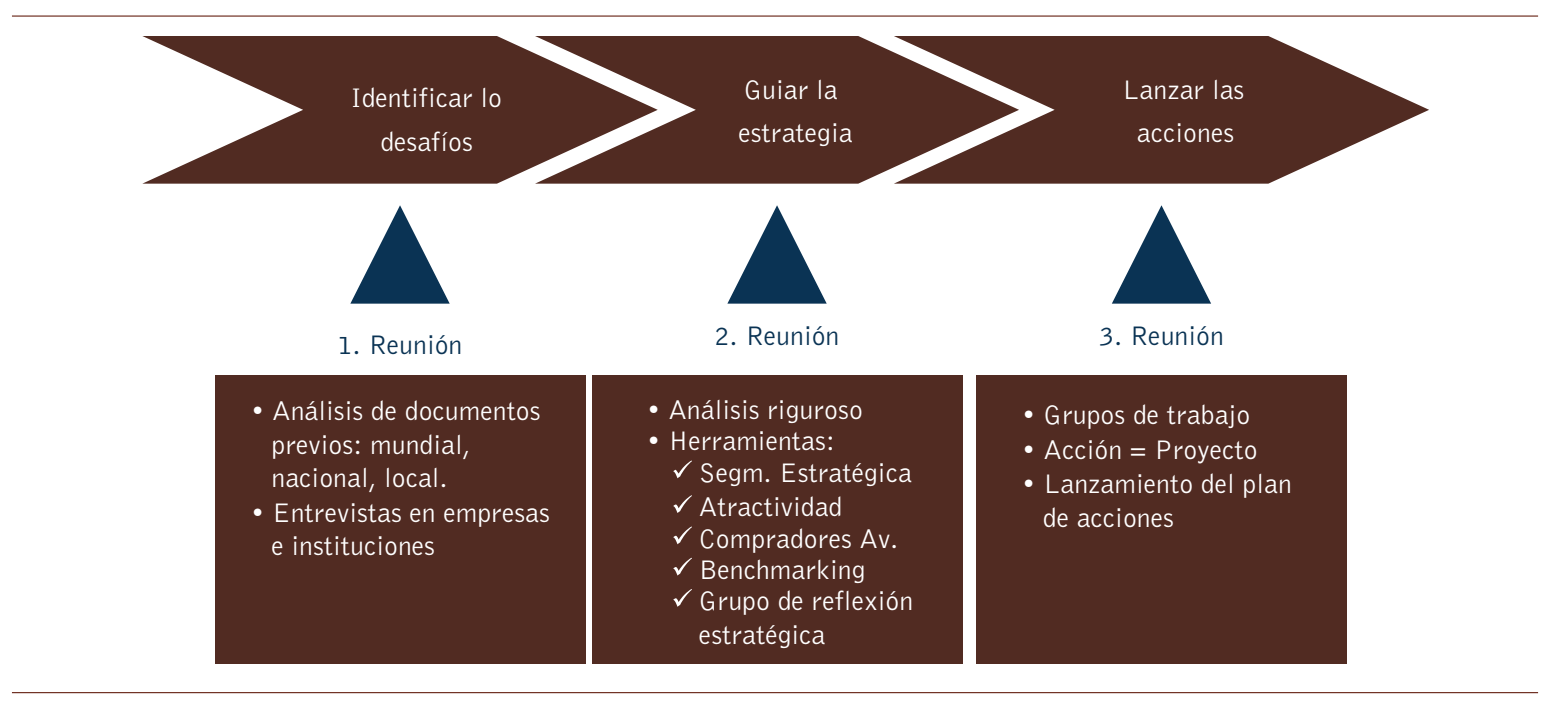

Fuente: Elaboración propia con base en Red Cluster Colombia (2014). 
la pertinencia de los programas respecto a las necesidades laborales de las empresas, y propender a un marco legal que incentive el desarrollo de áreas estratégicas como el turismo y la industria, principalmente para el Sistema Moda.

Otra variable relevante es el escaso uso de herramientas tecnológicas y de información para desarrollar estrategias de marketing digital que permitan promocionar productos $y$ servicios en redes sociales, sitios web y motores de búsqueda.

También se observó que los empresarios del clúster no se visualizan dentro del mismo, restringiendo la posibilidad de generar actividades de trabajo conjunto. Por el contrario, el desinterés por colaborar conlleva a un escenario de competencia depredadora que impacta negativamente en la oferta y calidad de servicios, ante la reducción de los márgenes de ganancia.

En el caso de la iniciativa Mi Destino Salud se pudo determinar que pese a ser un sector relativamente joven, el turismo médico es uno de los sectores más dinámicos de la economía global en la actualidad. Según cifras de la firma McKinsey \& Company (2009), el número de turistas médicos en el mundo está cercano a los 8 millones.

La tendencia es creciente, pero de igual forma lo es la cantidad de gobiernos de países que se interesan por impulsar políticas orientadas a desarrollar el turismo médico para convertir esta actividad en una nueva fuente de ingresos para sus economías. Cabe resaltar los casos de Turquía, India, Costa Rica y Estados Unidos, que, gracias a sus particularidades, desarrollan una ventaja competitiva que les facilita brindar un importante valor agregado a las personas que viajen a realizarse procedimientos o tratamientos médicos.

El turismo de salud surge como una respuesta a diferentes factores demográficos y socioeconómicos (Gráfico 2).
En el caso colombiano, desde el año 2008, el gobierno colombiano ha asumido la función de convertir el turismo médico en un sector de clase mundial. Sin embargo, se debe analizar detalladamente la oferta del sector hacia el mercado internacional, así como las capacidades de los agentes para adaptarse a las exigencias de los clientes extranjeros.

El desarrollo del turismo médico en Colombia aún es incipiente. Según cifras de McKinsey \& Company (2009), tan solo el $2,2 \%$ de los turistas que ingresan al país son turistas de salud. Sin embargo, la tendencia de crecimiento evidencia un potencial significativo para el desarrollo del sector en los próximos años (Gráfico 3).

De aproximadamente 35.000 turistas de salud que Ilegan a Colombia cada año, el $33 \%$ tiene como destino Bogotá, mientras que Cúcuta solo recibe el 3\%, tal como se puede observar en el Gráfico 4.

Por otro lado, en el clúster del sistema moda en la ciudad de Cúcuta, está comprendido por los sectores textil-confecciones-calzado y marroquinería, abarcando un outfit completo en cada colección. Según datos de la Cámara de Comercio de Cúcuta, el Sistema Moda cuenta con aproximadamente 2.000 empresas entre las que están fabricantes de tejido plano $60 \%$ y tejido de punto $40 \%$, fabricantes de calzado femenino, infantil, proveedores de insumos, comercializadores, gremios, entidades de apoyo y universidades.

La actividad que realiza este sector productivo está basada en el diseño y confección de prendas especializada en sport wear y casual wear, donde se identifican tendencias como jeans, alta costura femenina, casual masculino e infantil. En la parte de calzado y marroquinería, se fabrica en algunos casos de manera artesanal, dándole la característica de objeto único. Cuenta con profesionales y marcas reconocidas a nivel nacional y mundial, 
Gráfico 2. Factores asociados al turismo de salud

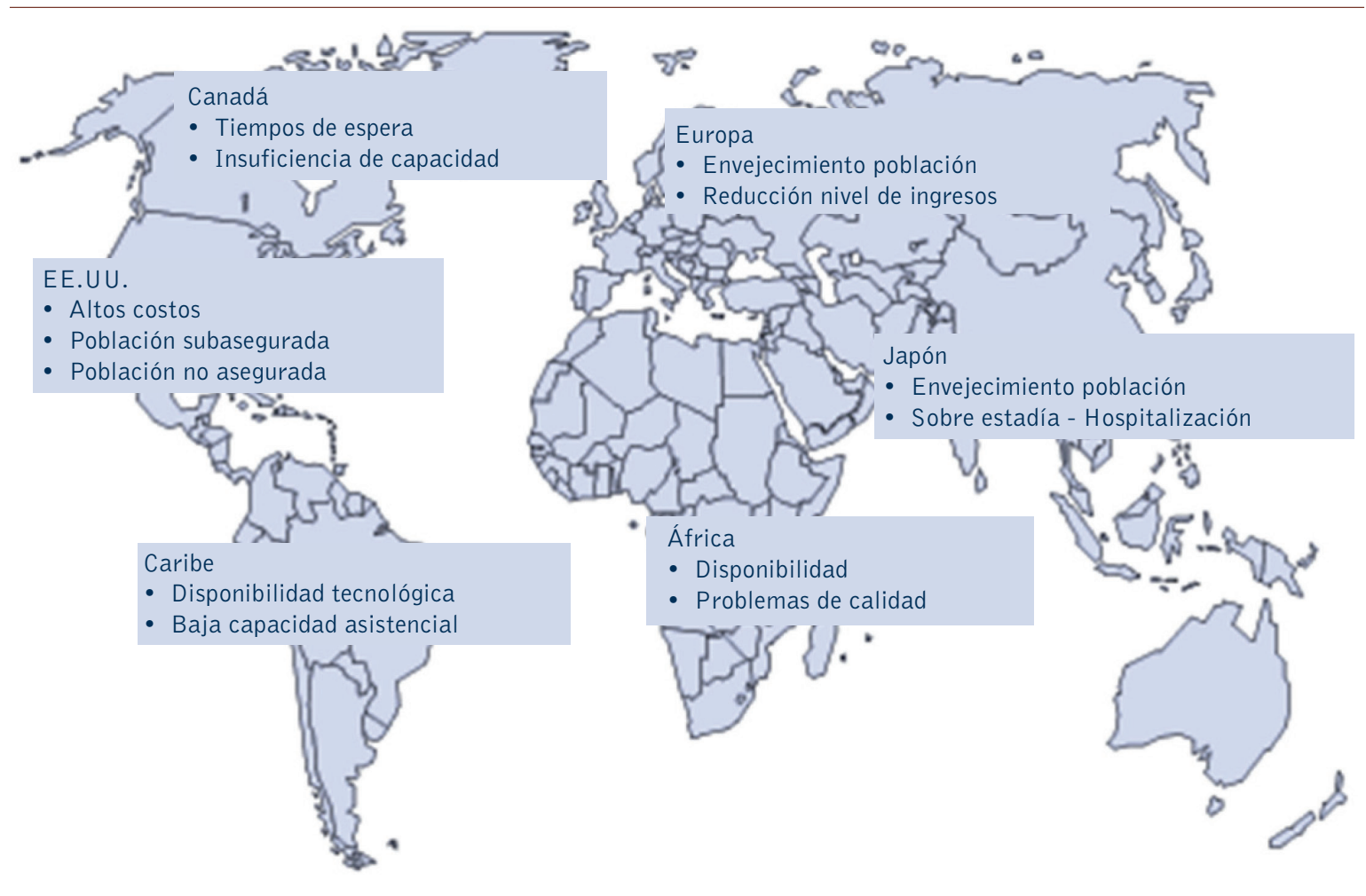

Fuente: Elaboración propia con base en Ministerio de Comercio, Industria y Turismo (2013).

Gráfico 3. Evolución del número de turistas de salud en Colombia (2010-2012).

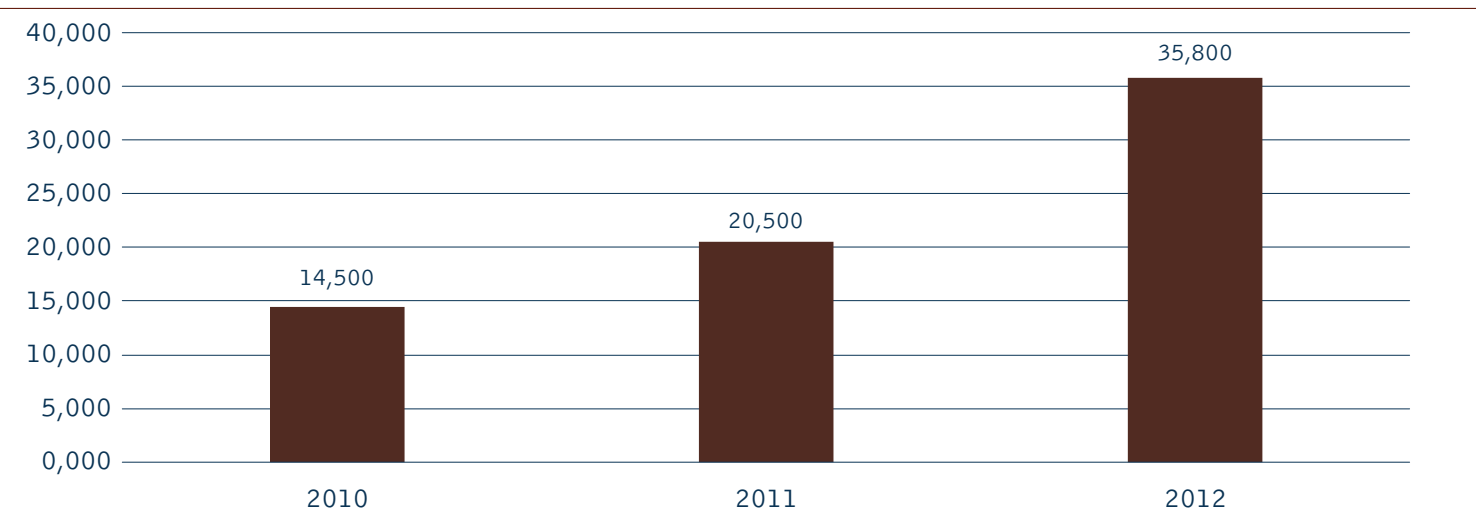

Fuente: Elaboración propia con base en Ministerio de Comercio, Industria y Turismo (2013).

identificándose con fortalezas como calidad, precio y servicio. Representantes importantes dentro del clúster son marcas como: Mussi Zapatos, Adriana Contreras, Lina Lemus, Khoor Jeans (Tanato), Grupo Nova, Creaciones Govelino, $A B C$ del Cuero y Gilvanny, entre otras marcas posicionadas en países como Estados
Unidos, Ecuador, Líbano, México, Perú, Nueva Zelanda, Panamá y Francia.

Sin embargo, el clúster está frente a un mercado exigente, en especial, los clientes internacionales, y de no ser competitivos no logran mantenerse en el mercado, a sabiendas de los beneficios que se tienen con los acuerdos 


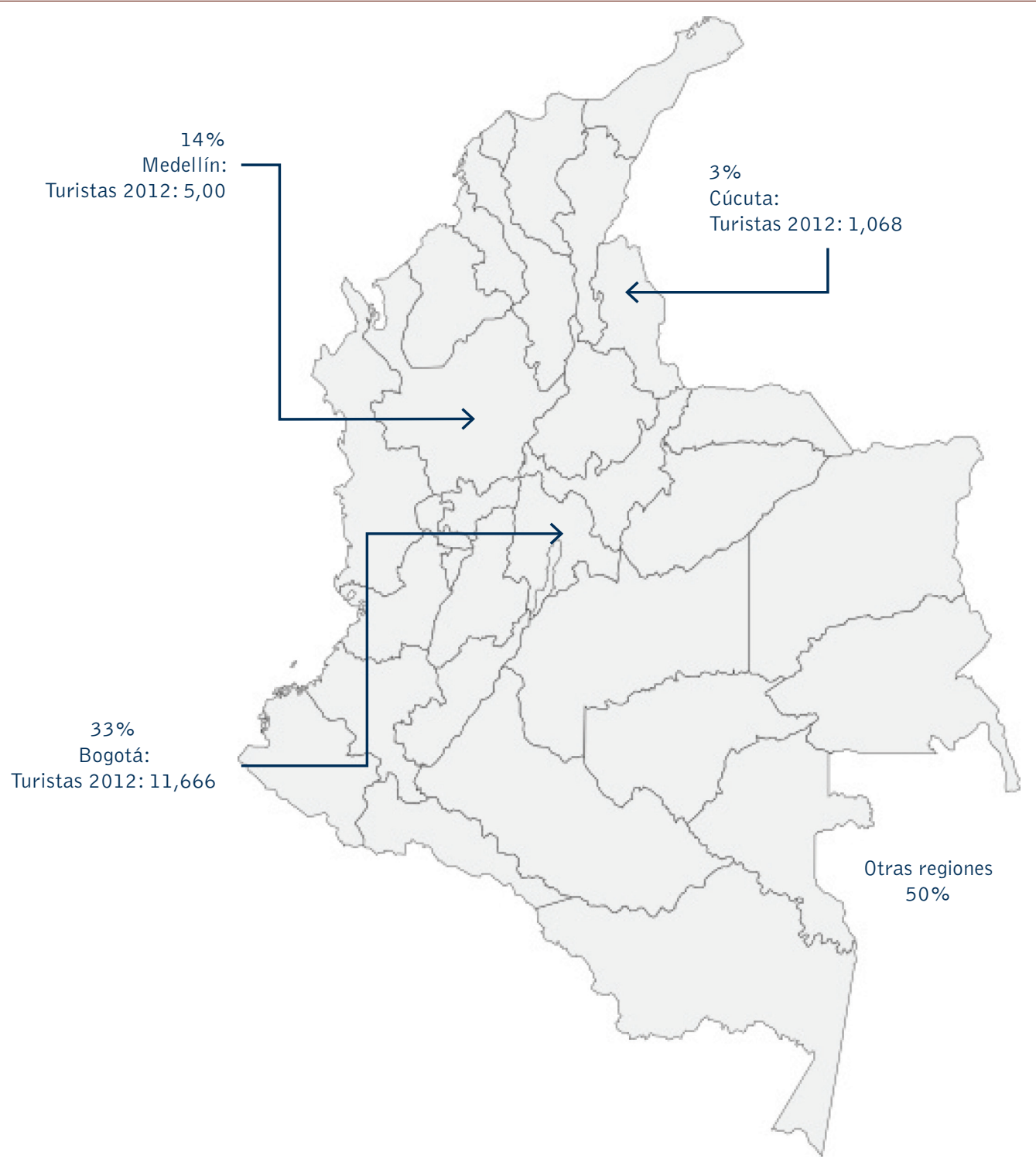

Fuente: Elaboración propia con base en Ministerio de Comercio, Industria y Comercio (2012).

de integración como los TLC. Sobrevivir a estos requisitos y precios de mano de obra no es fácil, por consiguiente, una estrategia en valor agregado es indispensable.

Según McKinsey (2014), Europa y Asia son los mercados más grandes del mundo en facturación, seguido de Estados Unidos; los países emergentes tienden a tener una mayor participación en el mercado, ya que, por ejem- plo, representan el $37 \%$ del total del segmento de mercado medio de mujeres y se espera que para 2025 alcance el 50\% del mismo.

En ambos casos, se encuentran sectores con alto potencial de crecimiento a nivel internacional, pero que exigen altos estándares para poder competir y que no se dan con facilidad en los empresarios de Norte de Santander, que tradicionalmente estuvieron acostumbrados al 


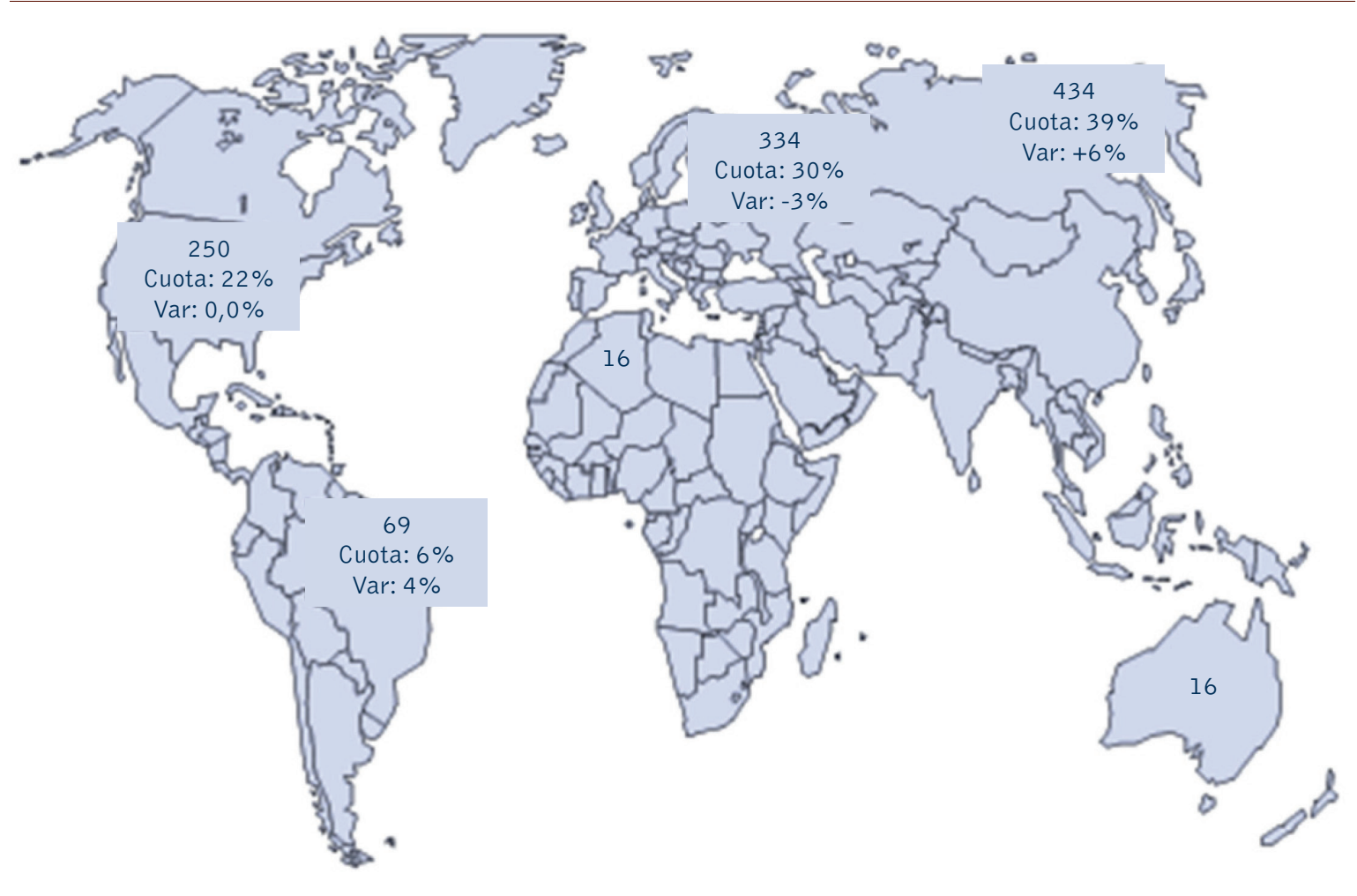

Fuente: Cálculos propios con base en McKinsey \& Company (2014).

mercado venezolano, sin sentir la necesidad de llevar a cabo actualizaciones o cambios significativos en las empresas.

Para la segunda fase, es pertinente explicar los resultados obtenidos de los viajes de referencia en el proceso de benchmarking.

\section{I BENCHMARKING EN COSTA RICA: REFERENTE PARA EL TURISMO DE SALUD}

Con el objetivo de realizar un benchmarking efectivo, se seleccionó Costa Rica, dados los elementos en común que se presentan el país centroamericano y Norte de Santander. Si bien en la actualidad hay diferencias sustanciales, antes de consolidarse el clúster de turismo médico en Costa Rica, este país enfrentó retos similares a los que hoy existen en Cúcuta y municipios aledaños.
La experiencia costarricense es una lección significativa, debido a las condiciones sobre las que se ha desarrollado el clúster, es decir, en un principio tuvieron barreras como el manejo de una segunda lengua, carencia de una cultura de servicios turísticos, entre otras variables que fueron superadas con el trabajo conjunto de diferentes agentes del clúster.

En la actualidad, Costa Rica es una plaza muy atractiva para pacientes originarios de Estados Unidos, ya que pueden acceder a servicios de calidad a un precio considerablemente inferior respecto al de su país. Sin embargo, en comparación con otros países de Centro y Sudamérica, las empresas costarricenses manejan precios más altos, principalmente por el costo de vida y el funcionamiento del dólar como moneda de libre circulación, junto con la moneda oficial que es el colón. 


\subsection{BENCHMARKING EN ESPAÑA Y HOLANDA: REFERENTE PARA EL SISTEMA MODA}

Con el viaje de referencia para visitar a empresas del sector de la moda en España y Holanda, se pudo validar el cambio en la dinámica del negocio de la moda, principalmente bajo el concepto de la moda rápida o pronta moda. Esto implica contar con una cadena de valor sincronizada y eficiente que permita reducir los ciclos de producción y distribución a períodos de dos semanas. En el caso de los proveedores de Zara, los volúmenes de pedidos que se manejan son altos y, por lo tanto, la capacidad de respuesta debe ser ágil.

La flexibilidad asume un rol importante en las empresas, que se complementa con la capacidad de generación de diseños, Ilegando en casos como el del proveedor de camisas para mujer, la empresa Happy Punt, que desarrolla más de 15.000 diseños al año, y son de uso exclusivo para Zara. Esto requiere que las empresas tengan equipos de diseñadores que sean capaces de interpretar las tendencias de los mercados para así desarrollar las nuevas colecciones.

Otro caso sobresaliente es el de Jean School en Ámsterdam, Holanda. Esta es la primera escuela dedicada de forma exclusiva a los procesos de diseño, manufactura y comercialización del jean. Esto se debe a que la ciudad ofrece una serie de ventajas para el negocio porque cuenta con elementos clave de la cadena de valor, como son el recurso humano calificado, centros de formación (Jean School), redes de cooperación (House of Denim), consultorías, eventos y ferias, infraestructura, tecnología y logística, que facilitan el desarrollo de los negocios para las empresas. Cabe resaltar que la mayoría de la producción se encuentra fuera de Holanda, pero el proceso de generación de valor agregado se desarrolla en Ámsterdam, procurando desarrollar un sector cada vez más innovador, sostenible y amigable con el medioambiente.

\subsection{LANZAR LAS ACCIONES}

Luego de realizar el benchmarking, se determinó que un factor relevante para que los clústeres se puedan consolidar es desarrollar productos y servicios orientados hacia los clientes, con alto valor agregado y especialización. Por ello, se formularon una serie de líneas de acción que permitieran impactar en aspectos fundamentales de la dinámica de los clústeres.

En el caso del turismo de salud:

- Desarrollo de centros de excelencia.

- Creación del club de producto Mi Destino Salud.

- Programa de marketing para el turismo de salud.

- Formación en pro del turismo de salud.

En el caso del Sistema Moda:

- Programa de formación en pro de la moda.

- Ciclo de conferencias.

- Velocidad de producción y logística.

- Gestión de la venta.

En ambos casos, las acciones se centran en la generación de valor agregado y el fortalecimiento de las capacidades del recurso humano. Igualmente, es prioritario generar un entorno propicio que fomente el emprendimiento y la innovación.

\section{CONCLUSIONES Y DISCUSIÓN}

Para que una estrategia de clusterización sea efectiva, se deben dar una serie de factores, entre los cuales se encuentran contar con 
certificaciones de calidad de nivel internacional como requisito desde el contexto. Por ejemplo, la certificación Joint Comission International - JCI, que identifica, mide y comparte con el mundo las mejores prácticas relacionadas con la calidad y la seguridad en la prestación de servicios médicos. Igualmente, la certificación Fairtrade, que respalda la implementación de las buenas prácticas empresariales y garantiza la distribución equitativa de los ingresos en la cadena de valor, dignificando el trabajo y promoviendo el respeto por el medioambiente.

Por otro lado, está la implementación de tecnología para incrementar la productividad. No se trata de tener tecnología por cumplir un requisito, porque esto implicaría lucro cesante: es contar con los equipos y la tecnología específica para el fortalecimiento de la competitividad.

Igualmente, es importante conformar equipos multidisciplinarios dispuestos a especializarse en un campo más estrecho. Acá, el médico es un miembro más del equipo de trabajo, y a él se suman otros profesionales de diferentes áreas formados y preparados desde su campo de conocimiento.

Desarrollar capacidades de gestión de datos y medición de resultados en tiempo real es otro factor importante, así como la transformación del aprendizaje en mejores protocolos para cada eslabón en la cadena de valor. En términos generales, una estrategia efectiva debe contar con una cadena de valor diferenciada, que permita incrementar el valor agregado a productos y servicios de cada clúster.

Por último, es vital para el avance de las iniciativas de clúster en Norte de Santander que se establezca con antelación si el liderazgo lo va a tomar el sector privado o público. Así mismo, debe seleccionarse muy bien el cluster manager, porque es la persona encargada de velar por el cumplimiento de los objetivos trazados en el clúster y su mejoramiento continuo.
Si bien las experiencias de clúster en Norte de Santander son recientes, hay aspectos como el del cluster manager y la financiación de la organización de cada clúster que tienen un impacto importante en eficiencia.

\section{REFERENCIAS}

Altés, C. (2006). El turismo en América Latina y el Caribe y la experiencia del BID (pp. 1-5, 18, 32). Banco Interamericano de Desarrollo, Washington, D.C.

Altvater y Mahnkop, B. (2002). Los límites de la globalización: Economía, ecología y política de la globalización. Siglo XXI-UNAM.

Bergmark, R.; Barr, D. y García, R. (2008), Mexican Immigrants in the us Living Far from the Border May Return to Mexico for Health Services. Journal of Immigrant and MinorityHealth, $12,610-614$

Gómez, A. (1999). Competitividad y clúster, de la teoría a la práctica. Gremial forestal de Guatemala. Disponible en: http://www.gremialforestal. com/articulos/competitividad_y_cluster_de_la_ teoria_a_la_practica.pdf

Jaramillo, R. (2003). Apuntes sobre los clusters turísticos. Turismo y Sociedad, 2.

Martínez, A. (2008). Hacia un nuevo sistema de la moda: el modelo Zara. Revista Internacional de Sociología (RIS), 66 (51), 105-122.

McKinsey \& Company (2009). Desarrollando sectores de clase mundial en Colombia. Sector Turismo de Salud. Ministerio de Comercio Industria y Turismo.

McKinsey \& Company (2010). Unleashing Fashion Growth City by City. Disponible en: http:// www.mckinsey.com/ /media/mckinsey/dotcom/ client_service/marketing \%20and\%20sales/pdfs/ unleashing_fashion_growth.ashx. Recuperado el 17 de septiembre de 2015.

McKinsey \& Company (2014). Succeeding in tomorrow's global fashion market. Disponible 
en: http://www.mckinseyonmarketingandsales.com/ succeeding-in-tomorrows-global-fashion-market. Recuperado el 12 de septiembre de 2015. Ministerio de Comercio, Industria y Turismo (2012). Documento Cluster Programa Rutas Competitivas 2013. Bogotá.

Ministerio de Comercio, Industria y Turismo (2013). Así avanza la transformación productiva. Programa de transformación productiva. Bogotá. Nalebuff y Brandenburger (1996). Coopetencia. Bogotá: Grupo Editorial Norma.
Nueno, J. L. (2001). "Examen a la moda". El País Semanal. Madrid, domingo 21 de marzo de 2001, pp. 152-158.

Porter, M. (2008). On Competition. Harvard Business Review Press.

Red Cluster Colombia (2014). Documento líneas de acción. Iniciativa, Mi destino salud. Cúcuta.

Vijaya, R. (2010), Medical Tourism: Revenue Generation or International Transfer of Healthcare Problems? Journal of Economic Issues, 44, 53-70. 